\title{
An Analytical Model for Deflection of Laterally Loaded Piles
}

Jen-Cheng Liao

Graduate Student, Department of Harbor and River Eng., National Taiwan Ocean University, Keelung, Taiwan, 20224.

\section{San-Shyan Lin}

Professor, Department of Harbor and River Eng., National Taiwan Ocean University, Keelung, Taiwan, 20224., sslin@mail.ntou.edu.tw

Follow this and additional works at: https://jmstt.ntou.edu.tw/journal

Part of the Civil and Environmental Engineering Commons

\section{Recommended Citation}

Liao, Jen-Cheng and Lin, San-Shyan (2003) "An Analytical Model for Deflection of Laterally Loaded Piles," Journal of Marine Science and Technology. Vol. 11: Iss. 3, Article 3.

DOI: $10.51400 / 2709-6998.2274$

Available at: https://jmstt.ntou.edu.tw/journal/vol11/iss3/3

This Research Article is brought to you for free and open access by Journal of Marine Science and Technology. It has been accepted for inclusion in Journal of Marine Science and Technology by an authorized editor of Journal of Marine Science and Technology. 


\section{An Analytical Model for Deflection of Laterally Loaded Piles}

\section{Acknowledgements}

This study was supported by the National Science Council, ROC, under grant number NSC 90-2211-E019-023. Grateful appreciation is expressed for this support. 


\title{
AN ANALYTICAL MODEL FOR DEFLECTION OF LATERALLY LOADED PILES
}

\author{
Jen-Cheng Liao* and San-Shyan Lin**
}

Key words: pile, lateral load, inclinometer, $p-y$ curve.

\begin{abstract}
ABATRACT
Load-deflection responses of laterally loaded piles are often obtained via in-situ lateral pile load tests. Problems of such tests are the difficulties occurring during data interpretation. In an effort to develop an efficient analytical method for analyzing deformation data of lateral loaded piles, an analytical model is proposed based on energy conservation of pile-soil system. The proposed analytical model not only provides a direct solution of the pile deflection function, but also has less need of complicated subsurface soil properties. In this paper, the authors present the theory and analytical model development of the proposed method. Two real cases are then used to verify the feasibility of the developed methodology as well as make comparison to other methods.
\end{abstract}

\section{INTRODUCTION}

The behavior of lateral loaded piles has attracted much attention in the past decade due to the increasing use of viaducts, marine oil boring platforms, and high rise building. While designing these structures, issues such as wind load, lateral spreading after soil liquefaction, and permanent horizontal ground movement occurred after seismic activities are very important and sometimes become critical condition of analysis. However, load-deflection responses of laterally loaded piles depend on many factors, such as pile geometry, structure material properties, adjacent soil conditions, soil-structure interaction, and loadings. Load-deflection responses of laterally loaded piles are often analyzed by using inclinometer data of lateral pile load tests. Problems of such tests are the difficulties occurring during data interpretation. In the past, complicated analytical models as well as numerical processes were

Paper Submitted 06/17/03, Accepted 09/01/03. Author for Correspondence: San-Shyan Lin.

* Graduate Student, Department of Harbor and River Eng., National Taiwan Ocean University, Keelung, Taiwan, 20224.

**Professor, Department of Harbor and River Eng., National Taiwan Ocean University, Keelung, Taiwan, 20224.

E-mail: sslin@mail.ntou.edu.tw developed to analyze such data. Available researches, such as by Scott [6], Ting [7], and Chiu and Chen [3], polynomial functions were used to simulate the deflection curve or the bending moment curve along the shaft. The coefficients of the polynomial functions are determined by fitting the data obtained from the inclinometer data. Brown et al. [1] and Brown and Zhang [2] proposed a method for interpretation of lateral load test. The method utilized a least squares regression technique that will converge to a solution for analytical $p-$ $y$ curves which produce minimum error between the predicted and measured deflection versus depth profile over a range of loading. The predicted deflection was obtained via the computer code COM624 [8]. In an effort to develop a time effective, simplified, and yet, accurate analytical method for analyzing deformation data of lateral loaded piles, the authors propose an efficient analytical model based on energy conservation of pile-soil system. In comparison to the present polynomial models of $p-y$ curves, the proposed analytical model not only provides a direct solution of the pile deflection function, but also has less need of complicated subsurface soil properties. In this paper, the authors present the theory and analytical model development of the proposed method. Two cases are then used to verify the feasibility of the developed methodology as well as make comparison to other methods.

\section{DEVELOPMENT OF DEFLECTION FUNCTION}

Study presented in this paper is in an effort to develop a time effective, simplified, and yet, accurate analytical method for analyzing deformation data of lateral loaded piles. The authors derived the deflection function of lateral loaded piles based on the energy conservation concepts with calculus of variation reduction applied. Inclinometer data of lateral loading pile test was then utilized to determine the coefficients of the derived actual deflection function. Once the deflection function is established, bending moment, shear force, and $p-y$ reaction at various depths along the pile can be reduced by differentiating the deflection function. 
Assumptions of the derivation include:

1. Soil deposits are idealized as nonlinear spring system;

2. Layered soil deposits can be represented by a series of spring system;

3. The pile material is assumed as homogeneous, isotropic and linearly elastic;

4. Lateral loading is applied only at ground surface where pile cap locates.

\section{Energy conservation- pile-soil interaction}

The total energy for a pile embedded in the soil medium is given as:

$$
\Pi=U+V
$$

Where $U=$ strain energy stored in the soil-pile system, and

$V=$ potential energy of soil-pile system caused by external loads.

As shown in Figure 1, the strain energy of the soilpile system can be expressed as:

$$
U=\frac{1}{2} \int_{0}^{L} E I\left(y^{\prime \prime}\right)^{2} d z
$$

Where $E=$ elastic modulus of the pile,

$I=$ moment of inertia of the pile,

$y=$ lateral deflection of the pile, and

$z=$ depth along the pile shaft.

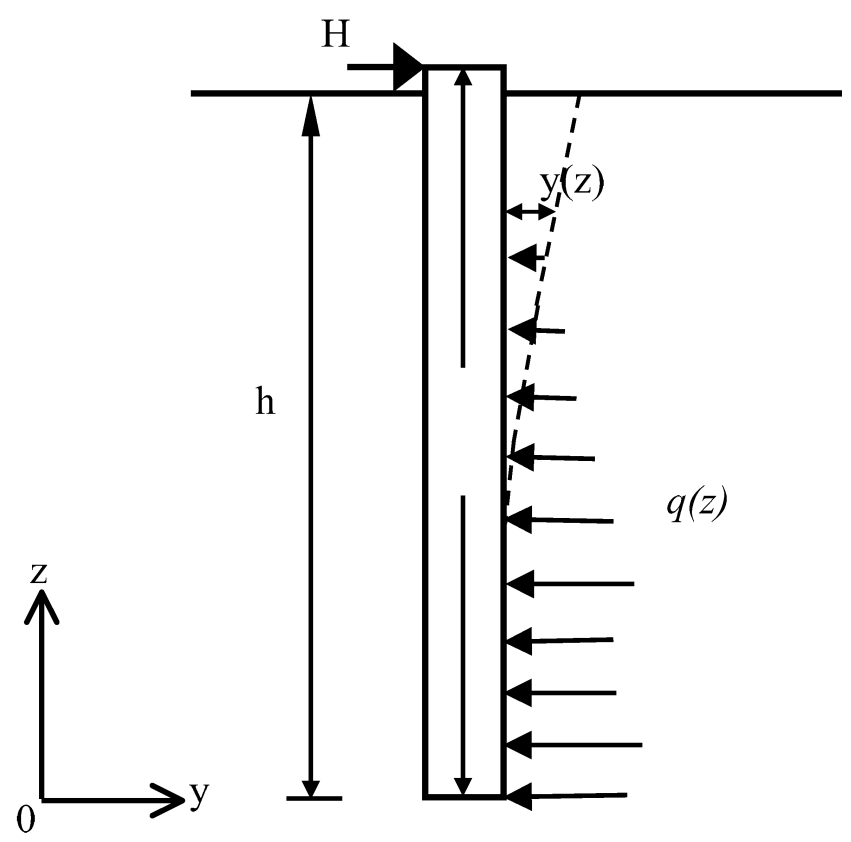

Fig. 1. Schematic of the soil-pile system.
The potential energy of the soil-pile system caused by external loading can be expressed as:

$$
V=\frac{1}{2} \int_{0}^{L} q(z) y d z-H y(L)
$$

Where $q(z)=$ soil reaction at depth $z$,

$H=$ lateral forces applied at the pile head, $y(L)=$ pile deflection at the pile head, and $L=$ embedded length of the pile.

Substituting equations (2) and (3) into (1), the total potential energy of the soil-pile system can be presented as:

$$
\Pi=\frac{1}{2} \int_{0}^{L} E I\left(y^{\prime \prime}\right)^{2} d z+\frac{1}{2} \int_{0}^{L} q(z) y d z-H y(L)
$$

Subsequently, by applying the calculus of variation to Equation (4), following Euler-Lagrangian governing equation can be obtained:

$$
E I y^{I V}+q(z)=0
$$

\section{Boundary conditions and solution forms of deflection functions}

Two different boundary conditions of long piles are considered as shown in Figure 2. The first case considers free head and fixed toe condition, which represents long piles without caps (Figure 2a). The other case considers fixed with sway at head and fixed at toe, which represents long piles constrained with pile caps at top. By applying Rayleigh-Ritz method, general form of deflection functions that satisfies Equation (5)

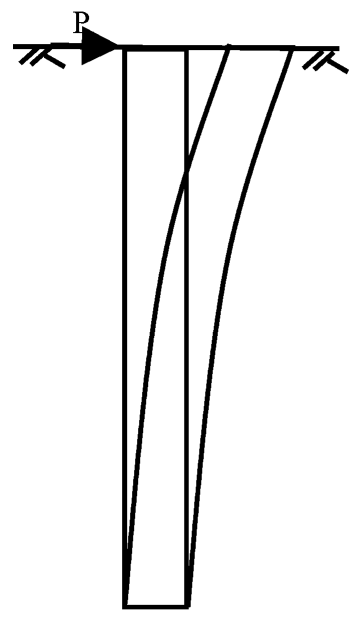

(a) Top-Free \& End-Fixed

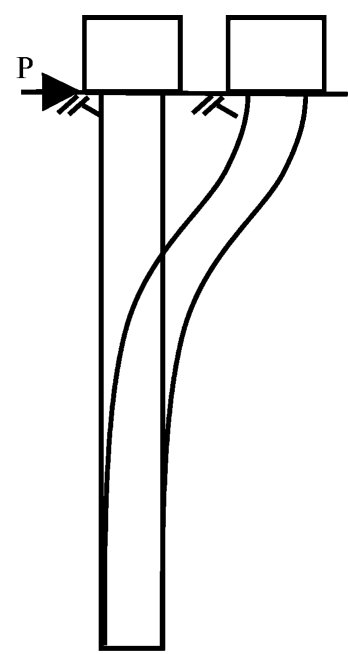

(b) Top-Fix with sway \& End-Fixed
Fig. 2. Different boundary conditions for laterally loaded piles. 
can then be determined as:

$$
y(z)_{i=1 \sim k}=\sum_{n=1}^{K} B_{n}\left(1-\cos \bar{N} \pi z_{i}\right)
$$

For top-free and toe-fixed boundary condition, $\bar{N}=\frac{2 n-1}{2 L}$

For top-fixed with sway and toe-fixed boundary condition, $\bar{N}=\frac{n}{L}$

Coefficients Bn can be solved by substituting Equation (6) into Equation (4), also by applying minimum potential theory $\left(\frac{\partial \Pi}{\partial B_{n}}=0\right)$. For the top-free and toefixed boundary condition, we have

$$
\begin{aligned}
E I B_{n} & \left(\frac{2 n-1}{2 L} \pi\right) \cdot \frac{1}{2} L+\frac{1}{2} E I\left[-2 B_{n}\left(\frac{2 n-1}{2 L}\right)^{3} \sin \left(\frac{2 n-1}{2} \pi\right)\right. \\
& +2 B_{n}\left(\frac{2 n-1}{2 L} \pi\right) \cdot \frac{1}{2} L-\sum_{n \neq m} B_{m}\left(\frac{2 n-1}{2 L}\right)^{3} \sin \left(\frac{2 n-1}{2} \pi\right) \\
& =P \\
& n, m=1, \ldots, k \\
& \quad \text { In addition, Equation (7) can also be expressed }
\end{aligned}
$$
into matrix form

$$
\mathbf{D} \cdot \mathbf{B}=\mathbf{P}
$$

The elements of matrix $\mathbf{D}$ an $\mathbf{P}$ are

$$
\begin{aligned}
& D_{n n}=E I\left(\frac{2 n-1}{2 L} \pi\right)^{4} \cdot \frac{1}{2} L-E I\left(\frac{2 n-1}{2 L}\right)^{3} \sin \left(\frac{2 n-1}{2} \pi\right) \\
& +E I\left(\frac{2 n-1}{2 L}\right)^{4} \cdot \frac{1}{2} L \\
& D_{n n}=\left(\frac{2 n-1}{2 L}\right)^{3} \sin \left(\frac{2 n-1}{2} \pi\right)
\end{aligned}
$$

and

$$
P_{n}=P \times L
$$

Similarly, for top-fixed with sway and toe-fixed boundary condition, we have

$$
\operatorname{EIB}_{n} L\left(\frac{n \pi}{L}\right)^{4}=P\left[1-(-1)^{n}\right)
$$

$B_{n}$ can be given as:

$$
B_{n}=\frac{P\left[1-(-1)^{n}\right)}{E I B\left(\frac{n \pi}{L}\right)^{4}}
$$

Based on beam theory, once the pile deflection function is determined, the moment, $M(z)$, the shear force, $V(z)$, the soil reaction, $q(z)$, of the pile along the shaft can be obtained as given in the following equations.

$$
M(z)=E I \frac{d^{2} y(z)}{d z^{2}} ; V(z)=\frac{d M}{d z}, \text { and } q(z)=\frac{d V}{d z}
$$

\section{Determination of $B_{n}$ using inclinometer data-applica- tions in practice}

Although coefficients of reflection function $B_{n}$ can be determined using Equations (6), (8), and (11), yet difficulties often occur in determining the value of optimized term number $k$ and lacking of soil reaction distribution $q(z)$. In a purpose to formulate the developed method into a practical engineering solution, the authors proposed to regress $B_{n}$ from the inclinometer data. Advantages of using inclinometer data include less instrumentation cost, better soil profile correlation, and better simulation of the soil-pile energy conservation system.

To further expand Equation (6), computed deflection at depth $\mathrm{z}$ can be presented as Equation (13).

$$
y(z)_{i=1 \sim j}=[A]_{j \times k}\{X\}_{k \times 1}
$$

Where

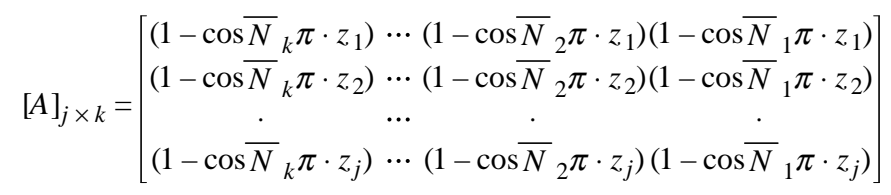

and

$$
\{X\}_{k \times 1}=\left\{\begin{array}{c}
B_{k} \\
B_{2} \\
B_{1}
\end{array}\right\} .
$$

The least square rule is then applied to perform regression to solve $\{X\}_{k \times 1}$ using procedures described below.

1. Obtaine the sum of the square of the error, $S$, between calculated and measured deflection (Equation 14).

$$
S=\{[A]\{X\}-\{Y\}\}^{T}\{[A]\{X\}-\{Y\}\}
$$

2. For minimum error, Equation 11 has to be satisfied and used to solve $\{X\}_{k \times 1}$ for optimized values of coefficients Bn.

$$
\left\{\frac{\partial S}{\partial x_{k}}\right\}=2[A]^{2}[A]\{X\}-2[A]^{T}\{Y\}=0
$$

The optimized of terms numbers are judged based on the regression analysis with suitable R-square values. 
Based on the authors experience, an analysis using eight terms often comes out good results.

\section{CASE STUDIES}

To verify the developed method, two cases of pile load tests were analyzed and compared to the results obtained using other methods. Results of the case studies are presented in the following paragraphs.

\section{Washington DOT lateral pile load test (Kramer, 1991)}

A full-scale lateral load test of pipe piles was

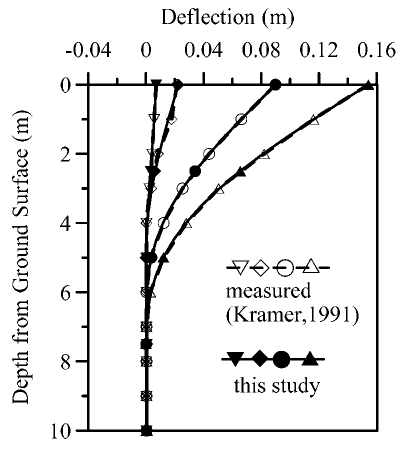

(a)

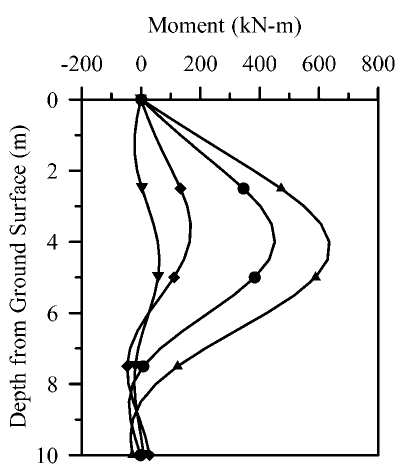

(c) (b)

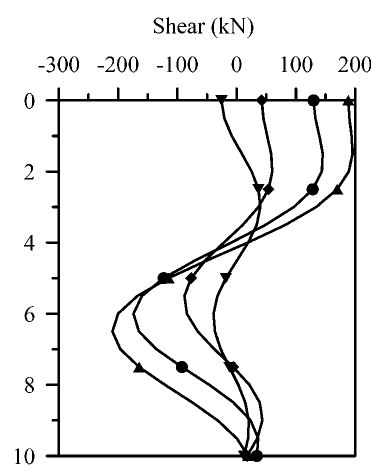

(d)
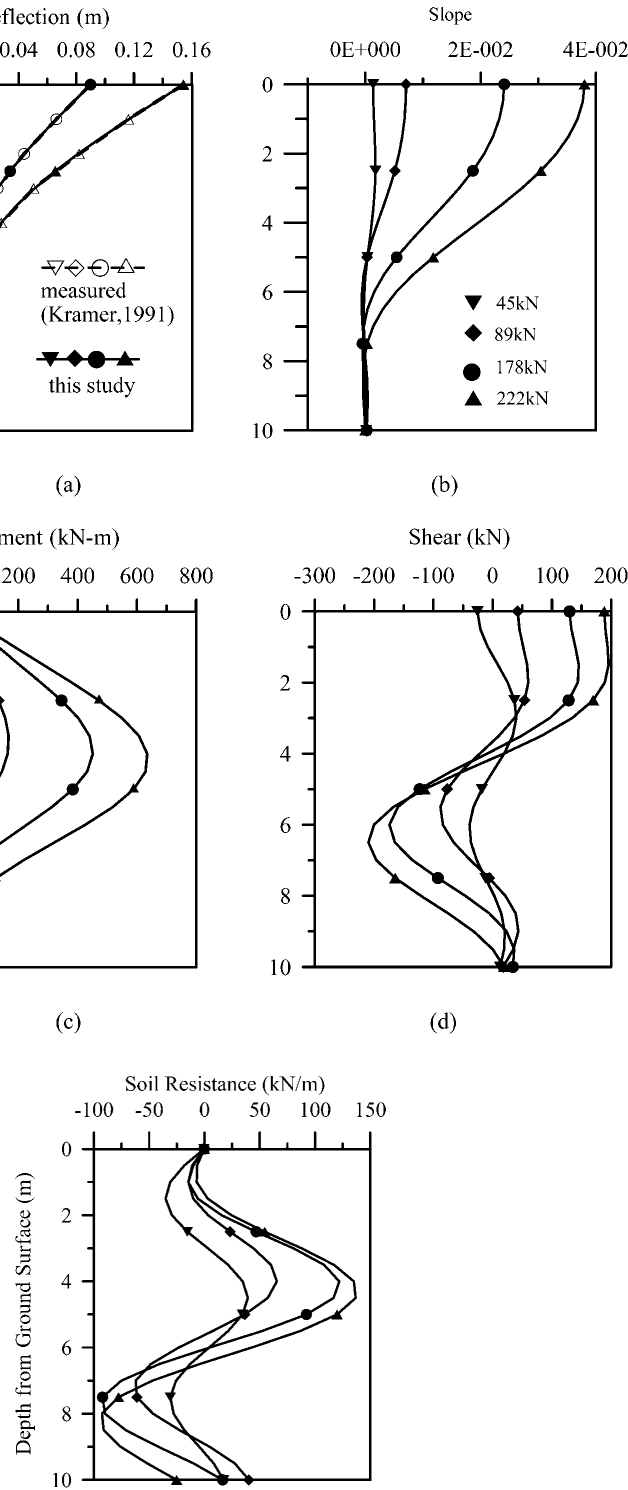

(e)

Fig. 3. Variations of deflection, deflection slope, bending moment, shear force, and soil resistance distributions along the pile shaft, WDOT Test Pile. conducted by Washington DOT and reported by Kramer [5]. The $24 \mathrm{~m}$ long pile, with diameter $D=0.476 \mathrm{~m}$, was embedded into deep deposit of soft clay and was subjected to $45 \mathrm{kN}, 85 \mathrm{kN}, 178 \mathrm{kN}$, and $222 \mathrm{kN}$ lateral load applied at head. Inclinometer measurements were obtained using an ingenious technique of lowering the casing into using an inflated air bag and only the deflection data can obtained. Top free and end fixed condition, as well as constant EI through entire pile length, are assumed when performing the proposed analysis.

Figure 3 shows the variations of deflection, deflection slope, bending moment, shear force, and soil resistance distributions along the pile shaft that were obtained from analysis. As shown in the figure, the maximum bending moment was found at $4 \mathrm{~m}$ below ground surface and the corresponding shear force is zero. Figure 4 shows the p-y curves obtained from the proposed method and derived by Brown et al. [1] at three different depths. The "Experimental deflection limit" represents the maximum measured deflection at the depth for the $p-y$ curve indicated. As indicated in the figure, the derived $p-y$ curve at $1.2 \mathrm{~m}$ depth shows that the soil resistance, $p$, increases with increase of deflection, $y$, up to a peak value. After passing the peak, the soil resistance decreases with further increase of deflection, and then reaches a constant residual value. For $p-y$ curves at depths of $3.0 \mathrm{~m}$ and $4.9 \mathrm{~m}$, the soil resistances increase as the deflection increases. Compare to the Brown's method, the proposed method provides a more simplified iteration process, and yet, reasonable prediction of the pile performance. Only basic spreadsheet functions are needed to perform such calculation instead of complicated computer program such as COM624 used by Brown et al. [1] and Brown and Zhang [2]. Figure 5 shows the calculated pile deflection at pile head comparing to the actual measurements. The differ-

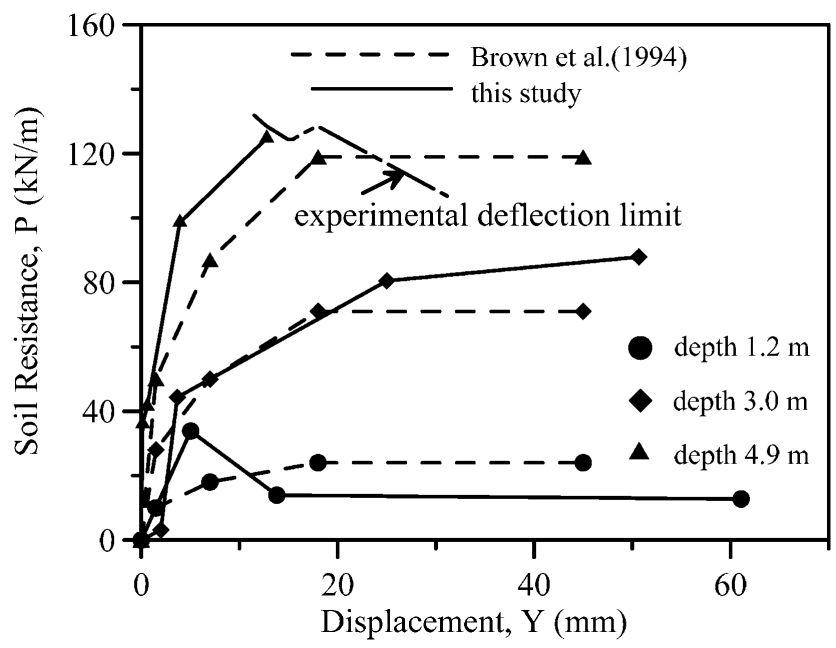

Fig. 4. $P-Y$ curve for WDOT Test Pile. 
ent were within $0.5 \%$ of the measure values.

\section{Lateral pile load test of H piles (Focht and Koch, 1973)}

Focht and Koch [4] reported a lateral load test of both single and group steel $H$ piles. Figure 6 shows the schematic layout of the test. The piles have a diameter $D=246 \mathrm{~mm}$; moment of inertia $I_{p}=8.74 \times 10^{7} \mathrm{~mm}^{4}$, and the elastic modulus $E_{p}=2 \times 10^{8} \mathrm{kPa}$. These piles are embedded in a stiff pile cap and are driven into a medium stiff clay deposit with an undrained shear strength of $72 \mathrm{kPa}$. Because Top fixed with sway and end fixed boundary condition was considered in the analysis for both single and group piles. Figure 7 show calculated and measured deflections and bending moment distributions of single pile and group piles, sub-

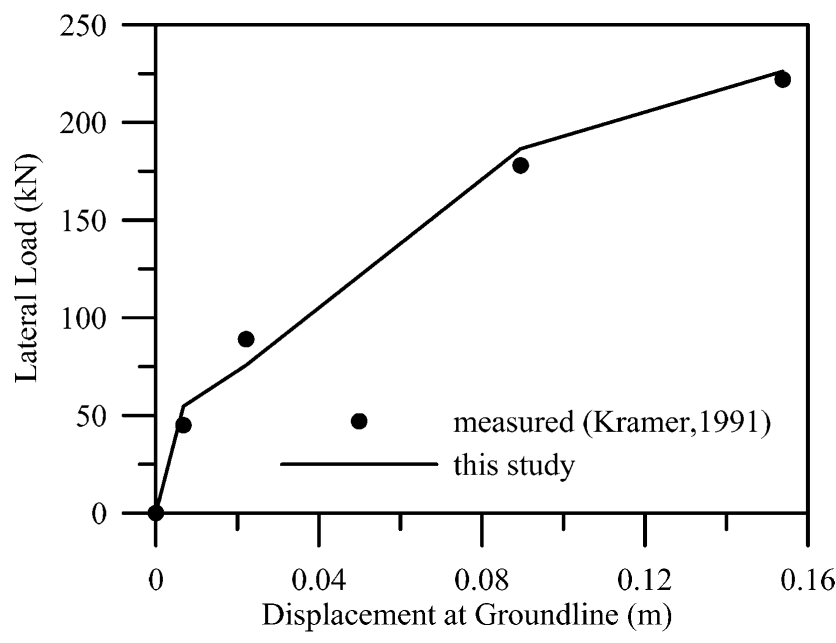

Fig. 5. Pilehead load versus deflection, WDOT Test Pile.

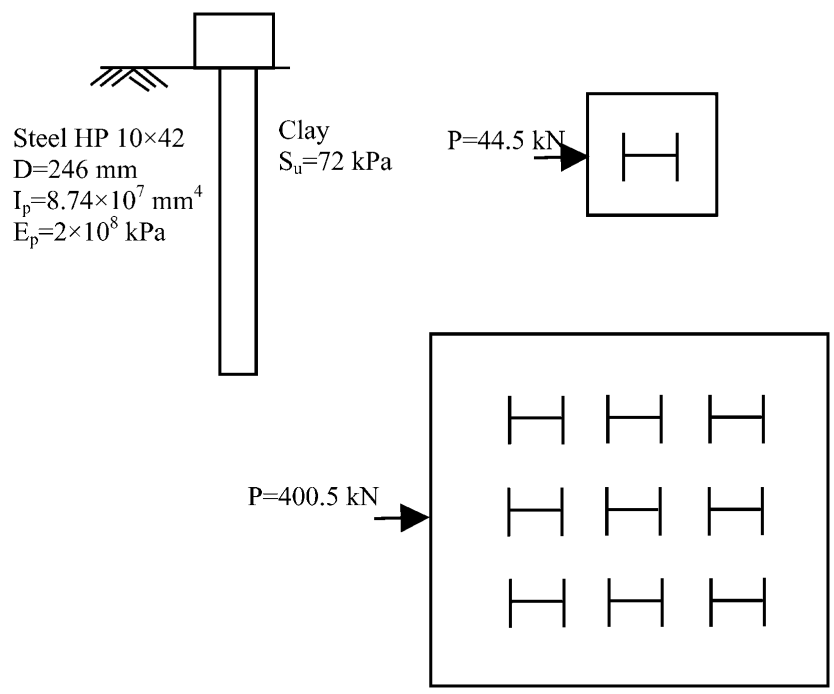

Fig. 6. Schematic layout of the test. jected to a lateral load of $44.5 \mathrm{kN}$ per pile. As shown in the Figure 7, the proposed method was able to successfully reproduce the deflection and to predict bending moment distribution in a reasonable agreement to the instrumentation measurements. The deflection of the group is twice the deflection of the single pile, and the maximum bending moment is about $20 \%$ higher, at the same load per pile. And the the deflection of any pile in a group causes movement of the surrounding soil and piles, thus leading to large deflection for the pile group than for single pile subjected to the same load per pile. The maximum bending moment in the group is larger than that for the single pile, because the soil allows the group to deflect more for the same load per pile, and the soil thus behaves as if it is soft. The proposed method slightly under-predict the bending moment of both single and group piles at top, possible reasons for this underestimation might be that some pile cap effects was neglected.

\section{CONCLUSIONS}

In this paper, the authors present a time effective, simplified, and yet, accurate analytical method for analyzing performance of lateral loaded piles. Background theories, as well as detailed derivation, of the proposed analytical method were described in this paper. Moreover, feasibility of the developed method was further verified using study results of two real case histories. Advantages of the proposed analytical method include:

1. This derived deflection function can provide a realistic description of relation between soil and piles.

2. Different boundary conditions of piles, such as fixed or free pile caps, can be easily adapted during the derivation of the deflection function, inefficiency of utilizing polynomials to fit deflection curves is no longer a problem.

3. Only inclinometer data is need for deriving the deflection function. Influence of surrounding soil de-
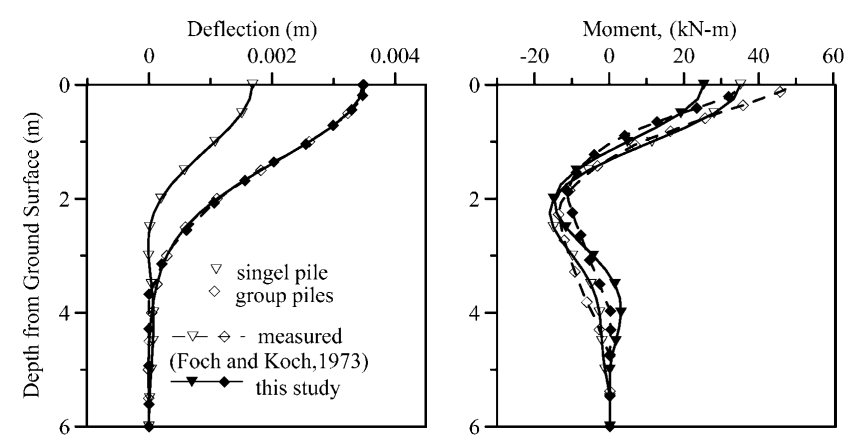

Fig. 7. Variations of deflection, bending moment, distributions along the pile shaft, H-Pile. 
posits and material properties of piles are considered when energy conservation of soil-pile system is satisfied.

4. Performance of both single and group piles can be analyzed using the developed model.

For short piles with high lateral loading, permanent lateral displacement might occur at bottom of piles. Definitions of strain energy and potential energy used in this study need to be modified in order to establish energy conservation relation. Energy conservation of soil-pile system can no longer be included using only inclinometer data. Further study is needed to develop the analytical model for the case of laterally loaded short pile.

\section{ACKNOWLEDGEMENT}

This study was supported by the National Science Council, ROC, under grant number NSC 90-2211-E019-023. Grateful appreciation is expressed for this support.

\section{REFERENCES}

1. Brown, D.A., Hidden, S.A., and Zhang, S., "Determination of P-Y Curves Using Inclinometer Data," Geotech. Testing J., 17(2), pp. 150-158 (1994).

2. Brown, D.A., and Zhang, S., "Determination of P-Y
Curve in Fractured Rock Using Inclinometer Data," International Conference on Design and Construction of Deep Foundations, FHWA, pp. 857-872 (1994).

3. Chiu, J.S., and Chen, C.H., "Regressive Analysis for Nonlinear Response of a Laterally Loaded Pile," J. Chinese Inst. Civil Hydraulic Engin., 12(3), pp. 455-464 (2000).

4. Focht, J.A., and Koch, K.J., "Rational Analysis of the Lateral Performance of Offshore Pile Groups," Proceeding of $5^{\text {th }}$ Offshore Technology Conference, Dallas, Tex, pp. 701-708 (1973).

5. Kramer, S.L., "Behavior of Piles in Full-Scale Field Lateral Loading Tests," Report No. WA-RD 215.1, Washington State Department of Transportation, USA (1991).

6. Scott, R.F., "Analysis of Centrifuge Pile Test: Simulation of Pile Driving," Report for the American Petroleum Institute OSAPR Project 13, California Institute of Technology, Pasadena, California (1980).

7. Ting, J.M., "Full-scale Cyclic Dynamic Lateral Pile Responses," J. Geotech. Engin., ASCE, 113(1), pp. 3045 (1987).

8. Wang, S.T., and Reese, L.C., "Analysis of Piles Under Lateral Load-COM624P for the Microcomputer," Report No. FHWA-SA-91-002, U.S. Department of Transportation, Federal Highway Administration, Washington, DC (1991). 